\title{
Shape Analysis Using the Edge-Based Laplacian
}

\author{
Furqan Aziz, Richard C. Wilson, and Edwin R. Hancock \\ Department of Computer Science, University of York, YO10 5GH, UK \\ \{furqan, wilson, erh\}@york.ac.uk
}

\begin{abstract}
This paper presents a novel analysis and application of the eigensystem of the edge-based Laplacian of a graph. The advantage of using the edge-based Laplacian over its vertex-based counterpart is that it significantly expands the set of differential operators that can be implemented in the graph domain. We commence by presenting a new mesh characterization based on the adjacency matrix of the mesh that captures both the geometric and topological properties of the shape. We use the edge-based eigenvalues to develop a novel method for defining pose-invariant signatures for non-rigid three-dimensional shapes based on the edge-based heat kernel. To illustrate the utility of our method, we perform numerous experiments applying the method to correspondence matching and classifying non-rigid three-dimensional shapes represented in terms of meshes.
\end{abstract}

\section{Introduction}

The key idea in the analysis of three-dimensional deformable shapes is to define an informative and discriminative feature descriptor that characterizes each point on the surface of the shape. Generally these techniques use a feature vector in $\mathbb{R}^{n}[12$, which contains both local and global information for that point. These feature descriptors can be used in many ways for analyzing threedimensional shapes. For correspondence matching, the descriptors are used to find potential correspondence among pairs of points on two different shapes [1]. For clustering the parts of a shape, the signatures can be used to identify semantically coherent parts of an object 34. Local descriptors can be combined in different ways to define a global shape signature and this can be used for shape classification or recognition $[5]$.

Recently, there is an increasing interest in descriptors obtained from the spectral decomposition of the Laplace-Beltrami operator associated with a shape. For example, Rustamov [3] has defined the Global Point Signature (GPS) that uses the spectrum of the discrete Laplace-Beltrami operator to represent threedimensional non-rigid shapes. Sun et al 2 have used a heat diffusion process to define signatures and this is referred to as the Heat Kernel Signature (HKS). Castellani et al [5] have used HKS to define Global Heat Kernel Signature (GHKS) and have used this for brain classification. Aubry et al[1] have proposed the Wave Kernel Signature (WKS) which represents the average probability of measuring a quantum mechanical particle at a specific location. All of these techniques use 
the spectrum of the discrete Laplacian, $\Delta$, an operator which is defined only on the vertices of a graph.

The discrete Laplacian defined over the vertices of a graph has found applications in many areas including computer vision and complex networks [7. However one of the limitation of discrete Laplacian is that it cannot link most results in analysis to a graph theoretic analogue. For example the wave equation $u_{t t}=\Delta u$, defined with the discrete Laplacian, does not have finite speed of propagation. In 89. Friedman and Tillich develop a calculus on graphs which provides a strong connection between graph theory and analysis. Their work is based on the fact that graph theory involves two different volume measures. i.e., a "vertex-based" measure and an "edge-based" measure. This approach has many advantages. Moreover it allows the direct application of many results from analysis to the graph domain.

Recently we have presented a new approach to characterizing points on a nonrigid three-dimensional shape 10 . This is based on the eigenvalues and eigenfunctions of the edge-based Laplacian, constructed over a mesh that approximates the shape. This leads to a new shape descriptor signature, called the Edge-based Heat Kernel Signature (EHKS). The EHKS was defined using the heat equation, which is based on the edge-based Laplacian. As first step we explored the application of the EHKS to shape segmentation. In this paper we take this study one step further. We use the EHKS for correspondence matching and show its robustness under noise. We also define a global signature (the GEHKS), which is based on the EHKS for shape classification. We perform numerous experiments and demonstrate the performance of the proposed methods on non-rigid three dimensional shapes and compare it to WKS.

\section{Edge-Based Eigensystem}

In this section we briefly review the eigenvalues and eigenfunction of the edgebased Laplacian $[9]$. Let $G=(\mathcal{V}, \mathcal{E})$ be a graph with a boundary $\partial G$. Let $\mathcal{G}$ be the geometric realization of $G$. The geometric realization is the metric space consisting of vertices $\mathcal{V}$ with a closed interval of length $l_{e}$ associated with each edge $e \in \mathcal{E}$. We associate an edge variable $x_{e}$ with each edge that represents the standard coordinate on the edge with $x_{e}(u)=0$ and $x_{e}(v)=1$. For our work, it will suffice to assume that the graph is finite with empty boundary (i.e., $\partial G=0$ ) and $l_{e}=1$.

The eigenpairs of the edge-based Laplacian can be expressed in terms of the eigenpairs of the normalized adjacency matrix of the graph. Let $A$ be the adjacency matrix of the graph $G$, and $\tilde{A}$ be the row normalized adjacency matrix. i.e., the $(i, j)$ th entry of $\tilde{A}$ is given as $\tilde{A}(i, j)=A(i, j) / \sum_{(k, j) \in \mathcal{E}} A(k, j)$. Let $(\phi(v), \lambda)$ be an eigenvector-eigenvalue pair for this matrix. Note $\phi($.$) is defined$ on vertices and may be extended along each edge to an edge-based eigenfunction. Let $\omega^{2}$ and $\phi\left(e, x_{e}\right)$ denote the edge-based eigenvalue and eigenfunction. Here $e=(u, v)$ represents an edge and $x_{e}$ is the standard coordinate on the edge (i.e., $x_{e}=0$ at $v$ and $x_{e}=1$ at $\left.u\right)$. Then the eigenpairs of the edge-based Laplacian are given as follows: 
1. For each $(\phi(v), \lambda)$ with $\lambda \neq \pm 1$, we have a pair of eigenvalues $\omega^{2}$ with $\omega=\cos ^{-1} \lambda$ and $\omega=2 \pi-\cos ^{-1} \lambda$. Since there are multiple solutions to $\omega=\cos ^{-1} \lambda$, we obtain an infinite sequence of eigenfunctions; if $\omega_{0} \in[0, \pi]$ is the principal solution, the eigenvalues are $\omega=\omega_{0}+2 \pi n$ and $\omega=2 \pi-\omega_{0}+$ $2 \pi n, n \geq 0$. The eigenfunctions are $\phi\left(e, x_{e}\right)=C(e) \cos \left(B(e)+\omega x_{e}\right)$ where

$$
\begin{gathered}
C(e)^{2}=\frac{\phi(v)^{2}+\phi(u)^{2}-2 \phi(v) \phi(u) \cos (\omega)}{\sin ^{2}(\omega)} \\
\tan (B(e))=\frac{\phi(v) \cos (\omega)-\phi(u)}{\phi(v) \sin (\omega)}
\end{gathered}
$$

There are two solutions here, $\left\{C, B_{0}\right\}$ or $\left\{-C, B_{0}+\pi\right\}$ but both give the same eigenfunction. The sign of $C(e)$ must be chosen correctly to match the phase.

2. $\lambda=1$ is always an eigenvalue of $\tilde{A}$. We obtain a principle frequency $\omega=0$, and therefore since $\phi\left(e, x_{e}\right)=C \cos (B)$ and so $\phi(v)=\phi(u)=C \cos (B)$, which is constant on the vertices.

3. If the graph is bipartite, then $\omega=-1$ is an eigenvalue of $\tilde{A}$. We obtain a principle frequency $\omega=\pi$, and then since $\phi\left(e, x_{e}\right)=C \cos \left(B+\pi x_{e}\right)$, so $\phi(v)=C \cos (B)=\phi(u)$ implying an alternating sign eigenfunction.

4. The sets $\{\pi+2 n \pi: n \geq 0\}$ and $\{2 \pi+2 n \pi: n \geq 0\}$ occur with multiplicity $|E|-|V|$. Note that although the eigenfunctions corresponding to these eigenvalues are zero on vertices, they are not zero on edge interiors.

This comprises all the principal eigenpairs which are supported on the vertices.

Note that although these eigenfunctions are orthogonal, they are not normalized. To normalize these eigenfunctions we need to find the normalization factor corresponding to each eigenvalue. Let $\rho(\omega)$ denotes the normalization factor corresponding to eigenvalue $\omega$. Then

$$
\rho^{2}(\omega)=\sum_{e \in \mathcal{E}} \int_{0}^{1} \phi^{2}\left(e, x_{e}\right) d x_{e}
$$

Evaluating the integral, we get

$$
\rho(\omega)=\sqrt{\sum_{e \in \mathcal{E}} C(e)^{2}\left[\frac{1}{2}+\frac{\sin (2 \omega+2 B(e))}{4 \omega}-\frac{\sin (2 B(e))}{4 \omega}\right]}
$$

Once we have the normalization factor to hand, we can compute a complete set of orthonormal bases by dividing each eigenfunction with the corresponding normalization factor. Therefore the orthonormalized eigenfunctions corresponding to eigenvalues $\omega^{2}$ are $\phi\left(e, x_{e}\right)=\frac{C(e)}{\rho(\omega)} \cos \left(B(e)+\omega x_{e}\right)$. Once normalized, these eigenfunctions form a complete set of orthonormal bases for $L^{2}(\mathcal{G}, \mathcal{E})$. 


\section{$3 \quad$ Shape Descriptors}

Once the edge-based eigenpairs are known, we can use them to link most of the results in analysis to the graph domain. Our goal is to use the solution of partial differential equations based on the edge-based Laplacian over a mesh for characterizing points on non-rigid 3D shapes. The signatures we propose here are based on the heat diffusion process governed by the equation

$$
\frac{\partial H_{t}}{\partial t}=-\Delta_{E} H_{t}
$$

where $\Delta_{E}$ is the edge-based Laplacian and $H_{t}$ is the heat kernel. The solution to above equation is called the heat kernel. The heat kernel has the following eigen-decomposition:

$$
H_{t}(x, y)=\sum_{i=0}^{\infty} e^{-\omega^{2} t} \phi(x) \phi(y)
$$

where $\left(\phi, \omega^{2}\right)$ are the edge-based eigenpairs.

\subsection{Local Descriptor}

Given a point $x$ on the surface of a three-dimensional shape, its Edge-based Heat Kernel Signature (EHKS) is given as 10]:

$$
\operatorname{EHKS}(x)=\left[H_{t_{0}}(x, x), H_{t_{1}}(x, x), \ldots, H_{t_{k}}(x, x)\right]
$$

In 10, we have experimentally shown the applications of EHKS for shape segmentation. In this paper we show the applications of EHKS for correspondence matching.

\subsection{Global Descriptor}

To extend our method to the problem of shape classification we define a global signature (GEHKS) for the whole shape which is based on the EHKS. Our approach of defining a global signature for the shape is closely related to the approach of [5]. Given a shape $S$, we define its global edge-based heat kernel signature as

$$
G E H K S(S)=\operatorname{hist}\left(\operatorname{EHKS}\left(x_{1}\right), \operatorname{EHK} S\left(x_{2}\right), \ldots, E H K S\left(x_{n}\right)\right)
$$

where hist(.) is the histogram operator. Since the GEHKS is defined on small and large values of $t$, it encodes both local and global information about the shape. 


\subsection{Discrete Settings}

A three dimensional shape can be conveniently represented by a mesh which approximates the shape. Therefore to find the corresponding edge-based Laplacian we need to find the adjacency matrix of the mesh. The simplest way of defining the adjacency matrix of the mesh using the un-weighted (0-1) or the weighted (distance or proximity) matrix is sensitive to the regularity of the particular triangulation and give little information about the shape itself. In [10] we have proposed a new method for for constructing the adjacency matrix of the mesh that uses the angle information between the edges and the area around each vertex (see Figure 1).

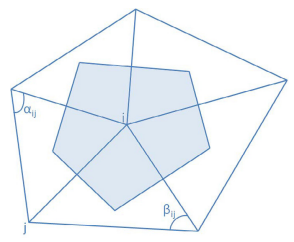

Fig. 1. Angles and the area appearing in the adjacency matrix

Let $M$ is a matrix whose $(i, j)$ th entry is defined as

$$
M(i, j)= \begin{cases}\frac{\cot \alpha_{i j}+\cot \beta i j}{2} & \text { if }(i, j) \in E \\ 0 & \text { otherwise }\end{cases}
$$

where $\alpha_{i j}$ and $\beta_{i j}$ are the angles opposite to the edge $(i, j)$, as shown in Figure 1 Let $\mathrm{S}$ be a diagonal matrix whose ith diagonal entry is the area associated with the triangles abetting the vertex $i$. We define the symmetric adjacency matrix as $A=S^{1 / 2} M S^{1 / 2}$. The $(i, j) t h$ entry of the adjacency matrix, in terms of the elements of the matrices $\mathrm{M}$ and $\mathrm{S}$, is given as follows 10 :

$$
A(i, j)= \begin{cases}\sqrt{S(i, i) S(j, j)} M(i, j) & \text { if }(i, j) \in E \\ 0 & \text { otherwise }\end{cases}
$$

The matrix defined above not only captures more information about the geometric and topological properties of the shape itself but also minimizes the dependence of the adjacency matrix on the mesh.

\section{Experiments}

In this section we will present both the qualitative and the quantitative analysis of the proposed edge-based heat kernel signature. We perform our experiments on the SHREC 2010 dataset, which contains 10 different shapes each with 20 
different non-rigid deformations. Figure2 shows some of these shapes. To find the edge-based eigenpairs, we first construct the adjacency matrix, as described in the previous section. We compute the area associated with each vertex using the method proposed in [1]. We then find the eigenpairs of the normalized adjacency matrix. To find signatures for shape we compute first 300 smallest eigenvalues and corresponding eigenvectors using the eigs routine in Matlab which is used to solve the sparse eigenvalue problem. We compute the scaled EHKS by uniformly sampling 100 points for different values of $t$ over the time interval $\left[t_{\text {min }}, t_{\text {max }}\right]$ where $t_{\min }=4 \ln 10 / \lambda_{300}$ and $t_{\max }=4 \ln 10 / \lambda_{2}[2$. In Figure $3(\mathrm{a})$ we have

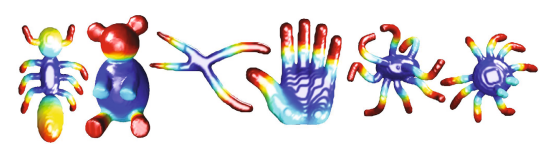

Fig. 2. The SHREC 2010 database of shapes

represented the values of EHKS with different colors on 6 different shapes of a human body, which shows the stability of the EHKS under different deformations of shapes. To prove the stability of our method, we illustrate the method on the problem of segmenting and classifying parts of a human body using EHKS. We select points on hands, feet, and head of 15 different poses a human body and compute their EHKS. To visualize the results, We apply PCA on these signatures and embed them in a three dimensional space. Figure $3(\mathrm{~b})$ shows that EHKS can not only distinguish between different classes of features, it can also distinguish classes of features of different shapes.

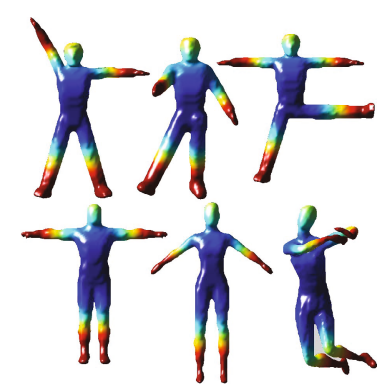

(a) EHKS for six shapes

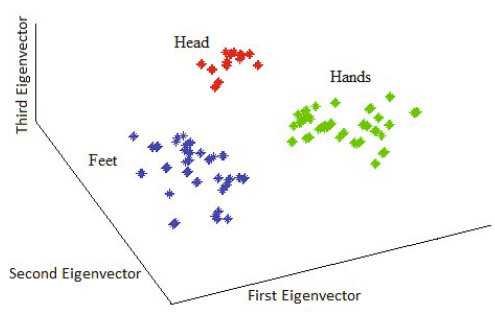

(b) Feature Points segmentation

Fig. 3. Performance of EHKS

In our next experiment, we show the stability of EHKS under controlled noise. For this purpose we take three-dimensional shapes of a human body and a bear 
and their deformed shapes. We add gaussian noise to the deformed shapes with mean $\mu=0$, and standard deviation $\sigma=0.3$. We select three different points on each of the given shape and compute their EHKS. Next we compute EHKS for each point on the deformed shapes corrupted by gaussian noise. We compute the Euclidean distance of the feature descriptor of the selected points with feature descriptor of each point on the deformed shape. In Figur 4 the lines between shapes show the first 50 best matches of each of the three points on the given shapes with the points on deformed shapes. Results show that the proposed method is robust under controlled gaussian noise. To demonstrate the usefulness

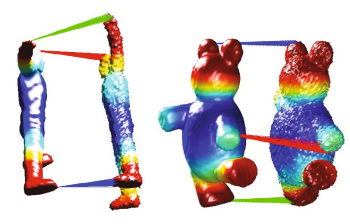

Fig. 4. Robustness under noise

of the proposed adjacency matrix, we compare the performance of the EHKS using different adjacency matrices. We select two different three-dimensional shapes of a human body. Next we randomly select a points on three different parts of the the shape and, for each point, find the first 50 best matches on the deformed shape. Figure 5 shows the results EHKS when computed from the proposed matrix(left), the matrix $A=\left(\frac{P+P^{T}}{2}\right)$ where $P=S^{-1} M$ (middle), and the symmetric matrix $M$ that uses the angle information only(right). Results shows that EHKS constructed using the proposed adjacency matrix is more stable. To

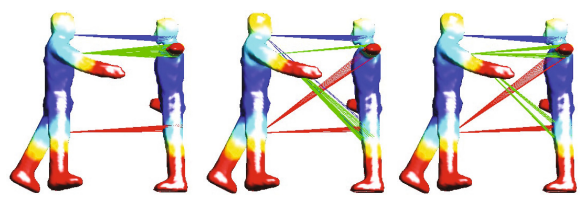

Fig. 5. Comparison of EHKS on different adjacency matrices

evaluate the performance of the proposed feature descriptor for correspondence matching, we select three-dimensional shapes of a human body, ant and glasses. We also select a deformed shape corresponding to each of these shape. For each three-dimensional shape we select a random point on five different parts of the shape and compute EHKS for each of these points. Next We compute the Euclidean distance of the feature descriptor of selected points on each shape with the feature descriptors of each point on the corresponding deformed shape. Figure 6(a) shows the first 50 best matches of each of the point on the shape with the 
points on deformed shapes. We perform a similar experiment for WKS(Figure 6(b) . Results show that EHKS is more robust and stable as compared to WKS. To compare the performance of EHKS with WKS, we select a three dimensional

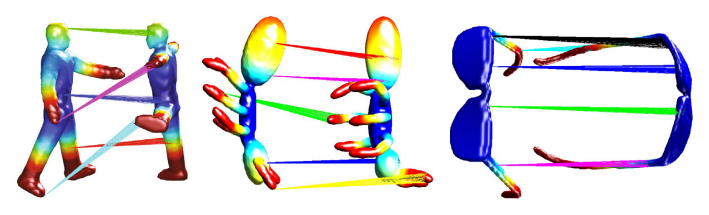

(a) EHKS on three different shapes
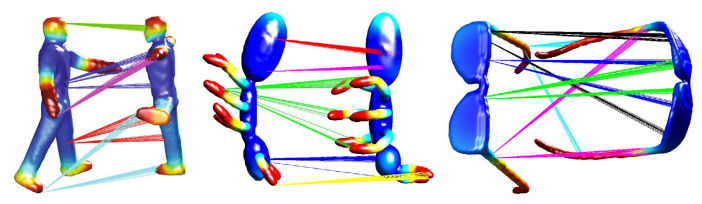

(b) WKS on three different shapes

Fig. 6. Comparing EHKS and WKS

shape of a human body and its deformed shape. we randomly select a point on 10 different parts of the shape and find the best match for each point on the deformed shape using both the EHKS and WKS. We repeat this experiment for five times. The number of successful matches for both methods are given in table 1.

Table 1. Number of best matches

\begin{tabular}{|l|l|l|l|l|l|}
\hline & 1 & 2 & 3 & 4 & 5 \\
\hline EHKS & 8 & 8 & 8 & 9 & 8 \\
\hline WKS & 6 & 8 & 7 & 7 & 8 \\
\hline
\end{tabular}

In our final experiment, we show the applications of GEHKS for shape classification. For this purpose, we select three-dimensional shapes of ant, plier and octopus from SHREC 2010 dataset with all of their deformations and compute GEHKS for each of these shapes. To visualize the results, We apply PCA on these GEHKS and embed them in a three dimensional space. Figure 7 shows that the proposed method can be useful for clustering different shapes. To compare the accuracy of the proposed method we perform a similar experiment with WKS and compute the rand indices for both methods. The accuracy of the proposed method was 0.8514 while the that of WKS was 0.7893 . These results show that the EHKS is more informative than the WKS, and gives higher performance for correspondence matching and shape classification. 


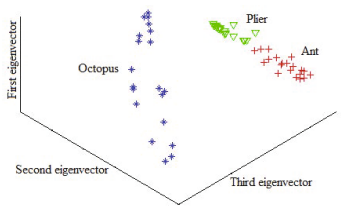

Fig. 7. Clustering of different shapes

\section{Conclusion and Future Work}

We have presented a method for analyzing three-dimensional non-rigid shapes which is based on heat equation defined over the edge-based Laplacian. Experimental results show that our method can be used for clustering, correspondence matching and classifying $3 \mathrm{D}$ shapes. In future, we would like to use the solutions of other partial differential equations over graph defined using the edge-based Laplacian, which have close relation to equation in analysis.

Acknowledgements. Edwin Hancock was supported by a Royal Society Wolfson Research Merit Award.

\section{References}

1. Aubry, M., Schlickewei, U., Cremers, D.: The wave kernel signature: A quantum mechanical approach to shape analysis. Tech. rep., TU München, Germany (2011)

2. Sun, J., Ovsjanikov, M., Guibas, L.: A concise and provably informative multi-scale signature based on heat diffusion. Comp. Grarph Forum, 1383-1392 (2010)

3. Rustamov, R.: Laplace-beltrami eigenfunctions for deformation invariant shape representation. In: Eurographics Symp. on Geom. Processing, pp. 225-233 (2007)

4. Aubry, M., Schlickewei, U., Cremers, D.: Pose-consistent 3d shape segmentation based on a quantum mechanical feature descriptor. In: Proc. 33rd DAGM Symposium, Frankfurt, Germany (2007)

5. Castellani, U., Mirtuono, P., Murino, V., Bellani, M., Rambaldelli, G., Tansella, M., Brambilla, P.: A New Shape Diffusion Descriptor for Brain Classification. In: Fichtinger, G., Martel, A., Peters, T. (eds.) MICCAI 2011, Part II. LNCS, vol. 6892, pp. 426-433. Springer, Heidelberg (2011)

6. Osada, R., Funkhouser, T., Chazelle, B., Dobkin, D.: Shape distributions. ACM Transactions on Graphics, 807-832 (2002)

7. Estrada, E.: Characterization of 3d molecular structure. Chemical Physics Letters $319,713-718(2000)$

8. Friedman, J., Tillich, J.: Calculus on graphs. CoRR (2004)

9. Friedman, J., Tillich, J.: Wave equations for graphs and the edge based laplacian. Pacific Journal of Mathematics, 229-266 (2004)

10. Aziz, F., Wilson, R., Hancock, E.: Shape signature using the edge-based laplacian. In: International Conference on Pattern Recognition (2012)

11. Xu, G.: Discrete laplace-beltrami operator on sphere and optimal spherical triangulations. International Journal of Computational Geometry, 75-93 (2006) 